\section{Java Music Club: A Standard- ized Mutual Support Program for Adults with Cognitive Change}

\section{Patrick M Cunningham*}

College of Health, School of Social Work, University of Alaska Anchorage, Alaska, USA

\begin{abstract}
Introduction

The use of a standardized program, designed by a music therapist, for cognitively impaired adults to improve well-being and attenuate cognitive decline.

\section{Methods}

An experimental control group study with eight participants for 26 weekly sessions. Mental status, level of depression, quality of life, and caregiver burden were measured prior to and following completion of the study. Quality of life was achieved as measured by a process evaluation.

Results

The study participants experienced less cognitive decline and depression when compared to the control group. There was no difference in caregiver burden among both groups.

Discussion: Participation in a mutual support group using music therapy may diminish cognitive decline and depression.

Keywords: Music therapy; Mutual support; Quality of life; Standardized program; Well-being
\end{abstract}

\section{Introduction}

At the present time, Alzheimer's disease remains an incurable, fatal illness impacting 5.3 million people living in the United States and close to 48 million people worldwide [1]. In the early stages of the disease, most victims remain in the home and are cared for by family and/or friends. As the disease progresses, the need for care increases often resulting in the need for out of home care consisting of day care, assisted living, and nursing home care. As the need for care increases, the situation of placing the cognitively impaired adult at risk for harm increases.

*Corresponding author: Patrick M Cunningham, College of Health, School of Social Work, University of Alaska Anchorage, Alaska, USA, Tel: +1 9077866902; E-mail: pmcunningham@uaa.alaska.edu

Citation: Cunningham PM (2016) Java Music Club: A Standardized Mutual Support Program for Adults with Cognitive Change. J Alzheimers Neurodegener 2: 006.

Received: April 09, 2016; Accepted: June 21, 2016; Published: July 05, 2016

\section{Literature Review}

\section{Social isolation and depression}

When cognition declines, social isolation and depression often increases. Dr. John Cacioppo, a psychologist at the University of Chicago, has studied social isolation over a period of 30 years, and has found that feelings of loneliness result in an increase in more rapid cognitive decline [2]. Numerous studies have linked loneliness with depression and, particularly, when in out-of-home placements [3-6]. These mental health concerns arise across the full spectrum of care from nursing homes, assisted and retirement living, day care centers and home care. The recipient of care is often in a passive role of being provided for, and waiting for instruction of how to spend the day [7]. Activities are determined by the care giver, with the goal of keeping the person engaged. Often, little input from the recipient of care is solicited because, as the cognition deteriorates, and the ability of the person to communicate, understand information provided, and to independently take part in activities, decreases [8]. The person may often feel a lack of control over their lives, no meaningfulness in their daily activities, and frustration over the patronizing communication from their caregivers.

\section{Social Revolution in Care}

Kristine Theurer and her associates call for the need for a "social revolution" to improve the psychosocial care and quality of life in elder care [9]. They have drawn from the literature and point out the need for programming that result in peer support, engagement, and being productive, that will result in the person achieving a valued social identity rather than erosion. Resident Engagement and Peer Support (REAP) is the model proposed for developing interventions to draw upon the strengths of the participants, their becoming active participants in this process, and achieving more meaningfulness in their lives.

Among the current psychosocial programming provided to persons with cognitive impairment is therapeutic recreation intended to improve functional abilities, physical and mental health status, and quality of life $[10,11]$. Recreational activities to reduce passive behavior and prevent functional decline produced minimal effects and low participation rates [12]. Activities are often scheduled rigidly, and rather than being engaging, and creating connections among the participants, are more entertaining, patronizing, and distracting [13].

Maintaining a passive role and experiencing superficial relationships often results in the person feeling useless, and life having no meaning. This often progresses to feelings of loneliness and depression [14]. It becomes increasingly difficult to assess loneliness and depression as cognitive impairment progresses. A sense of self is eroded; communication is difficult, resulting in behavioral responses ranging from silence to aggressive outbursts [15]. Needed are interventions that will result in moving from providing care to engagement of the person in meaningful relationships and activities. Active engagement of the elderly resulting in productive activity will change the view of the frail older person as an unproductive passive 
recipient of care [16]. Being productive may counteract the feeling of loneliness and depression. Elders who engage in activities encouraging mutual support among the participants may develop social relationships that increase their sense of well-being [17]. The support of peers who share a common experience may result in emotional bonding, despite reduced cognitive abilities [18]. It has positive consequences for mental health and reducing loneliness and depression $[19,20]$.

\section{Psychosocial interventions}

There have been a number of psychosocial interventions identified to counteract the negative effects of Alzheimer's and other related dementia, in order to improve the quality of life for adults currently diagnosed with the disease and at-risk populations that may experience the disease in the future. Assisting people with skills to remember information, memory recall, and direction draws upon cognitive training and therapy. The use of reminiscence taps a person's imagination and memories utilizing activities. For people living alone or in remote areas where direct assistance and contact is more difficult, various technologies were developed. To stimulate sensory dimensions, the use of touch and aroma is effective. The use of exercise and other activities are used to maintain health and to respond to challenging behaviors. Support groups offer a variety of potential benefits. Animal-assisted therapy has resulted in measurable positive effects on psychological and behavioral problems of dementia [21].

\section{Music therapy}

The use of music and music therapy with people who have dementia have received increased media attention in the past few years [22]. You Tube videos demonstrating the use of music and the movie Alive Inside depicted how the use of iPods can "awaken" people in the late stages of dementia where they become responsive to people and their surroundings, from an earlier state of withdrawal. Music may facilitate reminiscence, life review, validation, and reality orientation. When looking at the effect of music alone with how it affects dementia, studies are showing benefits from including music therapy in dementia care.

Spiro brings distinction, however, to the difference between simply including music in residential home settings and actively engaging residents in music therapy. A necessary element to improving the quality of life in residential settings is to increase activities residents are able to engage. The advantage of including music therapy in such settings is that it acts as a leisure activity for residents actively participating, improves memory and communication, and reduces behavioral difficulties adults with dementia may experience, such as depression, anxiety, and agitation. In distinguishing music therapy from typical musical inclusion in a residential setting, music therapy with adults who experience dementia, tends to involve participation in the music creation, such as the singing of recognizable songs, to trigger different parts of the brain. By using music to engage specific areas of the brain, memory deterioration can be reduced [23].

When working with adults who experience dementia, person-centered care is a necessary approach. Guidelines for ensuring person-centered care are being aware of communication pace and diction in addition to providing respect and dignity while learning about individual residents. The focus is upon improving their quality of life despite the predictable deterioration that occurs as dementia progresses [24].
A study examining the direct effects of music therapy towards agitation found that agitation was significantly reduced in the experimental group who participated in a music intervention program after six sessions, and continued for twelve sessions [25]. Further examining the effects of music therapy on the brain, the effects of music therapy were studied on how it impacted patients with frontal temporal dementia. Scores from the Neuropsychiatric Inventory Scale, Cohen Mansfield Agitation Inventory, and Cornell Scale for Depression in Dementia each decreased more than 50\% from the baseline demonstrating significant improvement in the quality of life. Disturbed behaviors such as persistent vocalizations, crying, wandering, and purposeless movements also declined by more than $50 \%$ from the baseline. Results from this study not only shows the direct impact of music therapy on emotional and behavioral functioning, but also suggests alternative treatments to potentially harmful or less effective medications [26].

A study looking at the effects of care givers and people with dementia who sang together in a pilot project showed a $90 \%$ engagement rating with improved quality of life scores from care givers. Rapport was improved between adults with dementia and their care givers while family members reported signs of the adults' original personalities [27]. A similar study for the effects of music therapy on patients and caregivers, noted improved attention, memory, use of language, interpersonal interactions, affection, and attitude [28].

A study linking sensory stimulation with person-centered care, found that engaging care takers in sensory activities increased care taker sensitivity towards their patients and promoted emotional well being in the patients which directly reflected on care givers. Thus, when reviewing the impacts of music therapy, especially in residential settings for adult dementia, using music therapy seems to provide benefits beyond direct brain activation. The quality of care may be improved by encouraging person-centered care through music therapy programs. Studies suggest that doing so, the quality of life improves for residents, patients, and family members alike [29].

Though limited, some research exists on the physical implications music therapy can have for adults with dementia outside of emotional and behavioral functioning. One concern with adults who have dementia is the occurrence of falling and having severe damage as a result. With their health already at risk from the disease, one study examined how music therapy could possibly act as an intervention to this occurrence due to its ability to improve motor functions. The best implications of this study would be to show how music therapy could reduce falls related to agitation. Falls were reduced from 12 months before the intervention to 3 months during the intervention, but it was difficult to determine if it was due entirely to music therapy. Therefore, more research would be required to gather statistically significant data. However, examining physical risk reduction in relation to music therapy is a meaningful area for future studies [30].

Five reasons why music stimulates brain activity [31,32]:

1. Music evokes emotions that bring memories.

2. Musical aptitude and appreciation are two of the last remaining abilities in dementia patients.

3. Music can bring emotional and physical closeness.

4. Singing is engaging.

5. Music can shift mood, manage stress and stimulate positive interactions. 
While there are multiple findings available regarding music therapy and its impact on cognitive functions, structured programs, and evaluations of those programs need to be developed to examine cost effective ways of intervention within residential homes. There are expected challenges to conducting research with Alzheimer's and dementia. Research participants may be difficult to obtain due to constraints of care takers working with adults experiencing dementia. Concerning family participation, members may feel embarrassed or upset by the demand for additional tests. These struggles seem to explain why many studies regarding music therapy with dementia tend to be smaller pilot studies. In conducting future research, these barriers will need to be challenged [33].

Music therapy is an established therapeutic intervention used to focus on cognitive, emotional, social, and physical needs of clients [34]. It may include singing, listening, creating, and moving to music. The intent is to build upon client's strengths and transfer to everyday life. Its use with clients who have dementia, has resulted in a growing body of evidence regarding its effectiveness in reducing troublesome behavioral symptoms such as withdrawal, agitation, aggression, and anxiety. In England, it has reduced the use of damaging anti-psychotic medication to control behavior [35,36]. A fundamental basis for the use of music therapy is that people communicate musically as a way of expressing feeling and relating to others.

\section{The Java Music Club}

The Java Music Club is the first standardized mutual support program addressing the critical rates of depression and loneliness across the senior living spectrum. It is a research-based wellness program for long-term care homes (including memory care), assisted living and retirement homes, adult day centers and community support centers. It was developed by Kristine Theurer, MA (Gerontology) MTA (Music Therapy), over a six-year period and implemented in 2010, in three nursing homes in British Columbia, Canada [37]. Ms. Theurer drew from her 20 years of experience as an accredited music therapist facilitating therapeutic groups. She found that a weekly discussion group centered around 52 themes, selected by participants, with carefully selected music related to the theme (142 songs in a songbook), along with pictures, affirmations, sayings and readings resulted in participants reporting benefits of reduced loneliness, understanding, mutual support, friendships, and increased coping skills [38]. Some of the theoretical foundation underlying this approach included Kitwood's concept of personhood and Riessman's helper therapy principle [39,40]. Personhood recognized a fundamental human drive to help others and, when inhibited, the person does not thrive. Helping not only helps the helped but also the helper. Those observing also receive benefits.

Some of the major objectives of the program are to:

- Develop mutual peer support

- Reduce loneliness and depression

- Increase social and emotional support

- Improve quality of life

- Prevent out of home placement

- Reduce caregiver burden

\section{Hypotheses and Methods}

This project is an experimental designed, random assignment, assessment of the participation effects of eight (8) clients for 26, one hour sessions, in a facilitated group program consisting of music, songs, theme pictures, affirmations, sayings, poetry, and interaction that will provide an opportunity for the participants to offer social and emotional support, experience well-being, and increase their quality of life. The site is the Salvation Army's Serendipity Adult Day Services located in Anchorage, Alaska. The research question is: Will participation in this program result in mutual support among the participants, increase their quality of life, and reduce the burden of their caregivers?

Hypothesis 1: Participation in the Java Music Club over a six-month period will result in mutual support.

Hypothesis 2: Participation in the Java Music Club over a six-month period will result in higher quality of life.

Hypothesis 3: Caregivers of participants in the experimental group will report less burden than caregivers in the control group.

Assignment to the control and experimental groups were random and drawn from the 55 clients who participate in the day care program. There were no major differences among both groups selected. This project is an assessment of the participation effects of eight (8) clients for 26, one hour sessions, in a facilitated group program consisting of music, songs, theme pictures, affirmations, sayings, poetry, and interaction that will provide an opportunity for the participants to offer social and emotional support, experience well-being, and increase their quality of life. Eight (8) clients were randomly selected for the control group.

Pre and post testing used the following instruments:

- Mini Mental Status Exam (MMSE)

- Reality Comprehension Clock Test (RCCT)

- Geriatric Depression Scale

- Alzheimer's Disease Related Quality of Life Scale (ADRQL)

- Zarit Burden Interview

The following measures were conducted at the end of the group sessions:

- Client Interview Schedule

- Facilitators Interview Schedule

- Directed focus group with participants

After each session (26) the following instrument was completed

- Process Measure: Mather Life Ways Institute on Aging Observing Quality of Life Scale

The session format is as follows:

\section{Preparation}

- CD player with background music, table with tablecloth, drinks, treats, chairs, and songbooks arranged

- The facilitator invites a group member to be an assistant and to choose the theme for the meeting

\section{Opening}

- Welcome to our meeting preamble is read

- Opening Song and ringing of chimes

- Group guidelines read followed by "getting centered"

- A song related to the theme is sung by the group 


\section{Middle}

Assistant is given "aboriginal talking stick" and asked to respond to one of the discussion questions related to the theme. The stick is passed from one member to the next

- A quote is chosen and the members are asked to take turns responding to it

- Another song is chosen and sung by the group using the song book

- A photograph is passed among the group, related to the theme, and members asked to respond to its meaning and collectively create a story

- Additional songs and quotes occur with members responding

\section{Closing}

- Closing affirmation read together

- Closing song "I will be there for you"

- Thank you handshake or hug offered

\section{Results}

The experimental group demonstrated a slight decline in their MMSE post-test scores compared to the control group who demonstrated a significant decline in MMSE scores (Table 1). There also were noticeable differences in the Geriatric Depression Scale. The experimental group significantly lessened to the amount of depression, whereas the control group showed a slight increase in depression. The other scales did not demonstrate significant differences among the groups.

The results of this pilot study demonstrated the value of the Java Music Club Program in providing the participants, referred to as guests, with the opportunity of sharing their feelings and providing mutual support to one another, adding to their quality of life. The evidence was obtained primarily from the process and post measures from the participants and staff. The process measure (Figure 1) observing the quality of life that was present during each of the 26 sessions, demonstrated pleasantness scores representing a clearly pleasant experience with moderate to high levels of activity. The highest score was in response to the question "Did the guest appear to enjoy the Java Music Club"? This was an extremely pleasant experience with a high level of activity. Selected comments from participants during the focus group were: "An opportunity to express my emotions and thoughts and get it out of my system." "I loved it, but wanted to meet more often." "We get to know each other much better." "It brought back a lot of different memories." "To join in this group is to forget my worries. It eases your problems. This is different, something special. It takes the loneliness away." A facilitator commented, "It has been an amazing and very rewarding experience. The approach has helped me know them better, and in a personal way, it has been a relaxing time with lots of fun." Another facilitator stated "It's my favorite group. I am amazed at how guests with dementia have responded... short, simple, yet profound answers".

Both mutual support among the participants and experiencing enhanced quality of life were observed during the 26 sessions to support hypotheses 1 and 2 . The third hypothesis where the caregivers reported less of a burden was not supported by the data.

\section{Limitations of Study}

The 26 sessions were intended to be weekly, but there was a two-month break due to staffing shortage, with 17 sessions remaining.

\begin{tabular}{|c|c|c|}
\hline & $\begin{array}{l}\text { Experimental (8) } \\
\text { Mean (sd) }\end{array}$ & $\begin{array}{l}\text { Control (8) } \\
\text { Mean (sd) }\end{array}$ \\
\hline Age, years & $71(11.8)$ & $74(10.2)$ \\
\hline Female to male ratio & $6: 02$ & $6: 02$ \\
\hline \multicolumn{3}{|c|}{ Mini Mental Status Exam (MMSE) } \\
\hline Pre-test & $19.3(6.9)$ & $21.1(3.6)$ \\
\hline Post-test & $18.8(5.6)$ & $17.9(5.9)$ \\
\hline t-test & 1.94 & $2.76^{*}$ \\
\hline \multicolumn{3}{|c|}{ Reality Comprehensive Clock Test } \\
\hline Pre-test & $2.6(1.3)$ & $3.1(1.2)$ \\
\hline Post-test & $2.2(.88)$ & $2.9(1.1)$ \\
\hline t-test & 1.42 & 1.54 \\
\hline \multicolumn{3}{|c|}{ Geriatric Depression Scale } \\
\hline Pre-test & $2.8(1.3)$ & $1.7(.95)$ \\
\hline Post-test & $1.5(1.0)$ & $2.0(1.0)$ \\
\hline t-test & $1.12^{*}$ & -1 \\
\hline \multicolumn{3}{|c|}{ Alzheimer's Disease Quality of Life Scale } \\
\hline Pre-test & $426.2(5.1)$ & $427.7(4.8)$ \\
\hline Post-test & $427.1(3.6)$ & $427.6(4.4)$ \\
\hline t-test & -0.715 & 0.008 \\
\hline \multicolumn{3}{|l|}{ Zarit Burden Interview } \\
\hline Pre-test & $25.2(4.2)$ & $29.2(6.3)$ \\
\hline Post-test & $26.6(3.9)$ & $28.7(6.1)$ \\
\hline t-test & -0.728 & 0.323 \\
\hline
\end{tabular}

Table 1: Demographic and mean scores of the standardized measurement instruments.

*indicates significance at 0.05 level

Also because the setting is a day treatment center, all eight of the members did not always attend each session. Caregiver burden measures may have also been affected by the break in session meetings.

\section{Discussion}

This pilot study demonstrated the value of the Java Music Club Program in providing the participants with the opportunity of sharing their feelings and providing mutual support to one another, adding to their quality of life. The pre and post testing findings were limited with only two significant differences found between the participants and controls. Participant's level of depression was less, as was increased cognitive impairment. Consistency in providing the program and attendance were also factors. The effects may have been greater if there wasn't the two-month break. Group process, when experienced on a more consistent basis, creates a positive synergy. It would have been interesting to determine if there were any differences among the participants, if frequency of attendance was measured. The strength of the findings was from the process measures, demonstrating an increase of quality of life and mutual support. The facilitators also experienced an increase in well-being and satisfaction from the Program. Clients highly valued the program and expressed a strong desire to continue it after the study was completed. Caregiver burden was anticipated to lessen because of the positive effects of the program, but consistency in attendance may have been a factor, as well as the short duration of the pilot project.

\section{Implications for Practice}

The use of the Java Music Club with cognitively impaired participants may prove to be a useful intervention in responding to 


\section{Pleasantness Scores}

\section{Sessions}

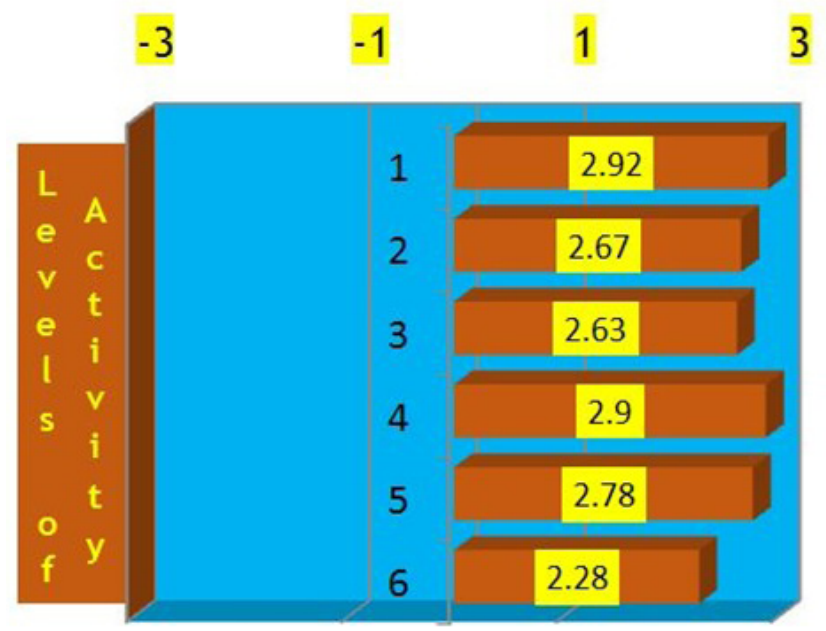

Figure 1: Pleasantness Scores.

1. Did the guest appear to enjoy the Java Music Club?

2. Did the guest sing along with the songs?

3. Did the guest participate in the discussion?

4. Did the guest appear to hear what was going on?

5. Did the guest use the song book?

6. Did the guest offer advice or support to others?

- Minus 3: An extremely unpleasant experience

- Minus 2: A clearly unpleasant experience

- Minus 1: A slightly unpleasant experience

- Zero: Neutral with no activity noted

- Plus 1: A slightly pleasant experience with low level of activity

- Plus 2: A clearly pleasant experience with moderate level of activity

- Plus 3: An extremely pleasant experience with a high level of activity

loneliness and depression, and encouraging mutual support and opportunity to improve quality of life resulting in well-being. Music therapy is beginning to be more recognized as an important psychosocial intervention to ameliorate a variety of symptoms in persons with cognitive challenges. Music and mutual support may decrease anxiety and agitation, thus reducing the need for psychotropic drugs for disruptive behavior.

\section{Conclusion}

This study demonstrated the effectiveness of using a structured, empathically designed music therapy program with cognitively impaired participants and achieving the goal of creating mutual support among the participants and improving their quality of life. The Day Care Center recognized the positive results obtained by the participants and have elected to include the program in its regularly scheduled activities. Future research will be conducted in assisted living and nursing homes where resident participation is more consistent.

\section{Declaration of Conflicting Interest}

The author declared no potential conflicts of interest with respect to the research, authorship, and/or publication of this article.

\section{References}

1. Alzheimer's Association (2015) 2015 Alzheimer's disease facts and figures. Alzheimers Dement 11: 332-384.

2. Cacioppo JT, Hawkley LC (2009) Perceived social isolation and cognition. Trends Cogn Sci 13: 447-454.

3. Bekhet AK, Zauszniewski JA (2012) Mental health of elders in retirement communities: is loneliness a key factor? Arch Psychiatr Nurs 26: 214-224.

4. Hagan R, Manktelow R, Taylor BJ, Mallett J (2014) Reducing loneliness amongst older people: a systematic search and narrative review. Aging Ment Health 18: 683-693.

5. Luanaigh CO, Lawlor BA (2008) Loneliness and the health of older people. Int J Geriatr Psychiatry 23: 1213-1221.

6. Cornwell EY, Waite LJ (2009) Social disconnectedness, perceived isolation, and health among older adults. J Health Soc Behav 50: 31-48.

7. Bartlett R, O'Connor D (2010) Broadening the dementia debate: Towards social citizenship. The Policy Press, Portland, Oregon, USA.

8. Alzheimer's Disease International (2013) Journey of Caring: An analysis of long-term care for dementia. World Alzheimer Report: 2013. Alzheimer's Disease International, London, England.

9. Theurer K, Mortenson WB, Stone R, Suto M, Timonen V, et al. (2015) The need for a social revolution in residential care. J Aging Stud 35: 201-210.

10. Leitner M, Leitner S (2012) Leisure Enhancement, (4 $4^{\text {th }}$ edn), Sagamore, Urbana, Illinois, USA.

11. American Therapeutic Recreation Association (2009) What is TR? American Therapeutic Recreation Association, Hattiesburg, Mississippi, USA.

12. Ayalon L, Gum AM, Feliciano L, Areán PA (2006) Effectiveness of nonpharmacological interventions for the management of neuropsychiatric symptoms in patients with dementia: a systematic review. Arch Intern Med 166: 21822188.

13. Wiersma E, Dupuis SL (2010) Becoming institutional bodies: Socialization into a long-term care home. Journal of Aging Studies 24: 278-291.

14. Kontos P, Martin W (2013) Embodiment and dementia: exploring critical narratives of selfhood, surveillance, and dementia care. Dementia (London) 12 : 288-302.

15. Behuniak SM (2010) Toward a political model of dementia: Power as compassionate care. Journal of Aging Studies 24: 231-240.

16. Brownie S, Horstmanshof $L$ (2011) The management of loneliness in aged care residents: An important therapeutic target for gerontological nursing. Geriatric Nursing 35: 318-325.

17. Kim S, Ferraro KF (2014) Do productive activities reduce inflammation in later life? Multiple roles, frequency of activities, and C-reactive protein. Gerontologist 54: 830-839.

18. Morhardt D, Spira M (2013) From person-centered care to relational centered care. Generations 37: 37-44.

19. Keyes SE, Clarke CL, Wilkinson H, Alexjuk EJ, Wilcockson J, et al. (2016) "We're all thrown in the same boat..." : A qualitative analysis of peer support in dementia care. Dementia (London) 15: 560-577.

20. Cruwys T, Haslam SA, Dingle GA, Jetten J, Hornsey JM, et al. (2014) Feeling connected again: Interventions that increase social identification reduce depression symptoms in community and clinical settings. Journal of Affective Disorders 159: 139-146.

21. Freeman M, Brown D, Janet Maier RN, O’Keeffe C, Wiener JM (2010) Innovative interventions for persons with Alzheimer's disease and their caregivers. ADSSP National Resource Center, Washington, D.C., USA.

22. Clements-Cortez A, Bartel L (2015) Sound stimulation in patients with Alzheimer's disease. Annals of Long-Term Care 23: 10-16. 
23. Spiro N (2010) Music and dementia: observing effects and searching for underlying theories. Aging Ment Health 14: 891-899.

24. Downs M (2013) Putting people and compassion first: The United Kingdom's approach to person-centered care for individuals with dementia. American Society on Aging 37: 53-59.

25. Lin Y, Chu H, Yang CY, Chen $\mathrm{CH}$, Chen SG, et al. (2011) Effectiveness of group music intervention against agitated behavior in elderly persons with dementia. Int J Geriatr Psychiatry 26: 670-678.

26. Raglio A, Bellandi D, Baiardi P, Gianotti M, Ubezio MC, et al. (2012) Music therapy in frontal temporal dementia: a case report. J Am Geriatr Soc 60: 1578-1579.

27. Camic PM, Williams CM, Meeten F (2013) Does a 'Singing Together Group' improve the quality of life of people with a dementia and their carers? A pilot evaluation study. Dementia (London) 12: 157-176.

28. Brotons M, Marti, P (2003) Music therapy with Alzheimer's patients and their family caregivers: A pilot project. Journal of Music Therapy 40:138-50

29. Lykkeslet E, Gjenedal E, and Storjord MB (2014) Sensory stimulation-A way of creating mutual relations in dementia care. Int J Qual Stud Health Well-being 9 .

30. Gill L, Englert N (2013) A music intervention's effect on falls in a dementia unit. The Journal for Nurse Practitioners 9: 562-567.

31. Maguire LE, Wanschura PB, Battaglia MM, Howell SN, Flinn JM (2015) Participation in active singing leads to cognitive improvements in individuals with dementia. J Am Geriatr Soc 63: 815-816.
32. Chu H, Yang CY, Lin Y, Ou KL, Lee TY, et al. (2014) The impact of group music therapy on depression and cognition in elderly persons with dementia: a randomized controlled study. Biol Res Nurs 16: 209-217.

33. Halpern A, peretz I, Cuddy L (2012) Introduction to special issue: dementia and music. Music Perception 29: 465-466.

34. American Music Therapy Association (2016) What is music therapy. American Music Therapy Association, Maryland, USA.

35. Gold K (2014) But does it do any good? Measuring the impact of music therapy on people with advanced dementia: (Innovative practice). Dementia (London) 13: 258-264

36. Wall M, Duffy A (2010) The effects of music therapy for older people with dementia. Br J Nurs 19: 108-113.

37. Theurer K (2010) The development and process evaluation of a co-led mutual support group in long-term care facilities 2010. Simon Fraser University, British Colombia, Canada.

38. Theurer K, Wister A, Sixsmith A, Chaudhury H, Lovegreen L (2014) The development and evaluation of mutual support groups in long-term care homes. J Appl Gerontol 33: 387-415.

39. Dewing J (1999) When your heart wants to remember: person centred dementia care. Nurs Stand 13: 4-22.

40. Riessman F (1965) The "helper therapy" principle. Social Work 10: 27-32. 